\title{
Structural Features Underlying Raloxifene's Biophysical Interaction with Bone Matrix
}

\author{
Nicoletta Bivi ${ }^{1}$, Haitao Hu ${ }^{1}$, Balagopalakrishna Chavali ${ }^{1}$, Michael J. Chalmers ${ }^{1}$, Christopher T. Reutter ${ }^{1}$, \\ Gregory L. Durst ${ }^{1}$, Anna Riley ${ }^{1}$, Masahiko Sato ${ }^{2}$, Matthew R. Allen ${ }^{2}$, David D. Burr ${ }^{2}$ and Jeffrey A. Dodge ${ }^{1}$ \\ ${ }^{1}$ Lilly Research Laboratories, Indianapolis, IN \\ ${ }^{2}$ Indiana University Purdue University Indianapolis, Indianapolis, IN
}

\begin{abstract}
Raloxifene, a selective estrogen receptor modulator (SERM), reduces fracture risk at least in part by improving the mechanical properties of bone in a cell- and estrogen receptor-independent manner. In this study, we determined that raloxifene directly interacts with the bone tissue. Through the use of multiple and complementary biophysical techniques including nuclear magnetic resonance (NMR) and Fourier transform infrared spectroscopy (FTIR), we show that raloxifene interacts specifically with the organic component or the organic/mineral composite, and not with hydroxyapatite. Structure-activity studies reveal that the basic side chain of raloxifene is an instrumental determinant in the interaction with bone. Thus, truncation of portions of the side chain reduces bone binding and also diminishes the increase in mechanical properties. Our results support a model wherein the piperidine interacts with bone matrix through electrostatic interactions with the piperidine nitrogen and through hydrophobic interactions (van der Waals) with the aliphatic groups in the side chain and the benzothiopene core. Furthermore, in silico prediction of the potential binding sites on the surface of collagen revealed the presence of a groove with sufficient space to accommodate raloxifene analogs. The hydroxyl groups on the benzothiophene nucleus, which are necessary for binding of SERMs to the estrogen receptor, are not required for binding to the bone surface, but mediate a more robust binding of the compound to the bone powder. In conclusion, we report herein a novel property of raloxifene analogs that allows them to interact with the bone tissue through potential contacts with the organic matrix and in particular collagen.
\end{abstract}

This is the author's manuscript of the article published in final edited form as:

Bivi, N., Hu, H., Chavali, B., Chalmers, M. J., Reutter, C. T., Durst, G. L., .. Dodge, J. A. (2016). Structural features underlying raloxifene's biophysical interaction with bone matrix. Bioorganic \& Medicinal Chemistry, 24(4), $759-767$. https://doi.org/10.1016/j.bmc.2015.12.045 


\section{Introduction}

Bone is a crucial tissue that provides protection to internal organs and support, as well as the ability to respond to environmental signals by adapting its geometry and mass. This unique combination of stability and adaptability is made possible by the composition of the bone matrix itself, where the mineral phase, the hydroxyapatite, provides stiffness, and the organic phase, mainly composed by collagen, provides the tensile strength. Moreover, throughout an individual's life, the bone matrix is constantly remodeled to meet metabolic and mechanical needs through the concerted effort of boneforming cells, the osteoblasts, and bone-resorbing cells, the osteoclasts.

Selective estrogen receptor modulators (SERMs) have been shown to decrease bone resorption and increase bone mineral density $[1,2]$ through direct interaction with the estrogen receptors followed by tissue selective transcriptional regulation [3]. Raloxifene is a clinically-validated SERM, which has been shown to mimic the protective effect of estrogen on the skeletal system without triggering the proliferative effects of estrogen on the breast and endometrium of the uterus [4,5]. Many clinical studies have confirmed the therapeutic effectiveness of raloxifene in reducing the risk of vertebral fractures in postmenopausal osteoporosis patients by significantly slowing bone turnover, preventing bone loss, and maintaining the structural integrity of cancellous bone [6-9].

The ability of raloxifene to reduce fracture risk is far greater than what would be predicted based solely on its effect on bone mineral density (BMD) $[10,11]$. Preclinical studies have documented that raloxifene leads to improved mechanical properties, most notably material-level toughness, despite little/no change in BMD or geometry/architecture [12-14]. Recent work has demonstrated that this effect of raloxifene is at least partly cell-independent [15]. Mechanistically, it has been proposed that raloxifene increases bound water within the matrix and this alters how strains are transferred between the organic and hydroxyapatite (HAP) portions of the matrix. Despite the biophysical evidence that this phenomenon is cell-independent, it remains unclear if this effect of raloxifene occurs through direct molecular interactions with bone and, if so, specifically what component of bone interacts with raloxifene.

Because a mechanism that is independent of the bone cells and the estrogen receptor (ER) pathway activation could represent an unexpected pharmacological approach to reducing osteoporotic fractures, we aimed to identify the structural features within the benzothiophene nucleus of the raloxifene that are responsible for this compound's interactions with bone tissue. We used multiple biophysical techniques including nuclear magnetic resonance (NMR) and Fourier transform infrared spectroscopy (FTIR) in order to quantify the interaction of benzothiophenes, estrogen and alendronate with bone and its components, and to investigate the molecular requirements that mediate that interaction. 


\section{Materials}

Ceramic Hydroxyapatite (HAP) Type I, $20 \mu \mathrm{m}$, was from BioRad, Cat\# 158-2000. 3D Collagen Culture System was from Millipore (Cat. No. ECM675). Poly D-Lysine 48-well plates used for the binding exposure studies were obtained from BIOCOAT (Cat. No. 354509, Lot No. 217048). DPBS (-Ca, -Mg) (Cat. No. 14-190-136) was from Fisher Scientific. Deuterated water $\left(D_{2} O\right)$ was from Sigma. SERMs (Table 1 ) were prepared as described [16,17]. The tested compounds were prepared as $10 \mathrm{mM}$ solutions in $100 \%$ DMSO or DMSO-d6 (Sigma Aldrich).

\section{Mechanical testing}

Skeletal tissue was obtained through a tissue sharing program at the Indiana University School of Medicine. All animals were on protocols that were approved by the Institutional Animal Care and Use Committee prior to their use. Femora from skeletally mature (15-24 mo/old) treatment naïve male hounds were machined into prismatic beams ( $\mathrm{N}=8-12$ beams per experimental group) following the details described previously [15]. Beams were subjected to at least one freeze-thaw cycle to ensure eradication of cellular activity. Absence of any cellular activity after a single freeze-thaw was demonstrated by lactate dehydrogenase immunostaining of fresh and frozen-thawed bone and the results have been published in [15]. Specified compounds were dissolved in dimethyl sulfoxide (DMSO) and then added to incubation media (1X PBS with 1\% penicillin-streptomycin supplementation) at a 2 $\mu \mathrm{M}$ concentration. Solutions were changed every other day over the course of 2 weeks. Control bones were soaked in PBS with $0.04 \%$ DMSO vol/vol ratio. At the conclusion of soaking, bone beams were subjected to four-point bending as previously described [15]. Energy to fracture was measured as the area under the force displacement curve. As these beams were machined to similar sizes, the parameter of energy to toughness is analogous to the modulus of toughness of the bone tissue as a material, described in previous work [15].

\section{Brunauer, Emmett and Teller (BET) Isotherms}

Bone tissue from animals was ground using a mortar and pestle to produce a powder that was then sieved using a wire mesh to produce a distribution of differently-sized particles $(<0.3 \mathrm{~mm},>0.3$ to $<0.53 \mathrm{~mm},>0.53$ to $<0.86 \mathrm{~mm}$, and $>0.86$ ). These batches were placed into $3 / 8$ inch diameter tubes and purged with nitrogen for 2 hours at $40^{\circ} \mathrm{C}$. The samples, equilibrated to room temperature, were analyzed with 7-point BET (partial pressures of $0.05-0.2$ ) using a Micromeritics Tristar 3000 with nitrogen adsorbate to assess surface area [18]. The batch with sample particle size of $<0.3 \mathrm{~mm}$ failed the canonical acceptance criteria that require the plot of BET value versus P/Po to have the linear regression of $r<0.9975$ due to an outlier in the data. This batch $(<0.3 \mathrm{~mm})$ was then tested on a Micromeritics ASAP 2020. Approximately 5 grams of this batch $(<0.3 \mathrm{~mm})$ sample were placed into a $1 / 2$ inch diameter surface tube and purged with nitrogen at $60^{\circ} \mathrm{C}$ for approximately 6 hours. After equilibrating to room temperature, the sample was analyzed with a 7 point BET, as described above, using a Micromeritics ASAP 2020 with krypton adsorbate. The results met the acceptance criteria $(r$ 
$>0.9975$ ) and are reported as BET SSA (Specific Surface Area). Total surface area (TSA) is calculated by multiplying the BET SSA result by the sample weight.

\section{Scanning Electron Microscopy (SEM)}

For SEM, powder samples were sprinkled on $13 \mathrm{~mm}$ SEM stubs with carbon adhesive tabs using a stainless steel spatula. Stubs were then tapped to remove excess sample and sputter coated with Au:Pd (gold palladium 60:40) target. FEI 200 Quanta ESEM Imaging was conducted in low vacuum mode, at $20 \mathrm{kv}$, with 3.0 spot size and magnifications of 50-2500x.

\section{Energy Dispersive Spectroscopy (EDS)}

SEM/EDS imaging was performed to assess the size heterogeneity of the bone powder and determine the most appropriate particle size to be used in further experiments. Bone powder $(<0.3 \mathrm{~mm}$ sample only) was rinsed gently with PBS (3X) and then divided into 2 batches. One batch was dried for $1 \mathrm{~h}$ in a vacuum oven at $37^{\circ} \mathrm{C}$ and then flushed with Argon. The other batch was air dried and flushed with Argon. Samples were sprinkled on $13 \mathrm{~mm}$ SEM stubs with carbon adhesive tabs using a stainless steel spatula. Stubs were then tapped to remove excess sample, but they were not sputter coated. SEM/EDS imaging was conducted at 15-25kv, with magnifications of 30-500x using a FEI 200Quanta ESEM [19].

\section{Nuclear Magnetic Resonance (NMR)}

A modified version of a protocol previously described [20] was used. In this version, a quantitative measurement is obtained, by calculating the integral of the NMR signal, which is directly proportional to the concentration of the compound in solution. Solutions of compounds $(50 \mu \mathrm{M})$ mixed in deuterated water $\left(D_{2} \mathrm{O}\right)$ were divided into two equal aliquots. One aliquot $(0.7 \mathrm{ml})$ was incubated with $<0.3 \mathrm{~mm}$ bone powder (10 mg) or HAP ( $5 \mathrm{mg}$ ) in a $1.5 \mathrm{ml}$ Eppendorf tube, for $1 \mathrm{~h}$ and $16 \mathrm{~h}$ under rotation at room temperature, while the other aliquot $(0.7 \mathrm{ml})$ was incubated without bone powder or HAP, but maintained under rotation at room temperature. After incubation, samples were centrifuged at 1,000 g for 5 minutes and the supernatant was transferred to a new tube and frozen at $-20^{\circ} \mathrm{C}$ until analysis. To evaluate binding to collagen, 3D collagen matrices (Merck-Millipore) were prepared following the manufacturer's instructions and $0.2 \mathrm{ml}$ of collagen was added to each well of a 48-well plate (Biocoat) and allowed to polymerize at $37^{\circ} \mathrm{C}$. Incubations were performed with $0.7 \mathrm{ml}$ of solution containing 50 $\mu \mathrm{M}$ of compounds (raloxifene, 88074 and 189005 ) at $37^{\circ} \mathrm{C}$ for $1 \mathrm{~h}$ or $16 \mathrm{~h}$. Control solutions were incubated in wells without polymerized collagen. After the indicated incubation times, solutions were aspirated with a pipette and stored at $-20^{\circ} \mathrm{C}$ until analysis. All NMR data were obtained using a Bruker Avance III $600 \mathrm{MHz}$ spectrometer equipped with a quadruple resonance $\left({ }^{1} \mathrm{H} /{ }^{19} \mathrm{~F} /{ }^{13} \mathrm{C} /{ }^{15} \mathrm{~N}\right) \mathrm{QCl}$ CryoProbe. Spectra were acquired with a standard 1D proton sequence. Residual water signals were suppressed with presaturation at a pulse power of $50 \mathrm{~Hz}$ during the relaxation delay. A $1.7 \mathrm{~s}$ acquisition time and a $2 \mathrm{~s}$ relaxation delay were used. The total number of transients ranged from 256 to 1024, with 4 dummy 
scans. The spectral width was $16 \mathrm{ppm}$. All data were processed using the standard Bruker software (Topspin). The raw data were zero-filled to $64 \mathrm{k}$ points and a $2 \mathrm{~Hz}$ line-broadening was applied before Fourier transformation.

\section{Fourier Transform Infrared Spectroscopy (FTIR)}

The infrared spectrum of $<0.3 \mathrm{~mm}$ bone powder alone, or bone powder exposed to $20 \mu \mathrm{M}$ raloxifene for $1 \mathrm{~h}$ was recorded over the $400-4000 \mathrm{~cm}^{-1}$ range with a Thermo Nicolet 5700 FTIR spectrometer. For the actual measurements, after an exposure experiment (as described above), the supernatants were discarded and the matrices (bone powder, HAP, or Collagen) were washed (1X) with $1.5 \mathrm{ml}$ of RO water, spun at $1000 \mathrm{~g}$ for $5 \mathrm{~min}$ and stored at $-20^{\circ} \mathrm{C}$. These matrices were then ground with ultra dry $\mathrm{KBr}(2 \mathrm{mg}$ sample/300 $\mathrm{mg} \mathrm{KBr}$ ) and thin pellets were obtained under vacuum. FTIR spectra were collected on these pellets. The background and sample spectra were averaged over 50 scans and with a resolution of $2 \mathrm{~cm}^{-}$ 1 .

\section{Computational Methods}

Lowest-energy conformations of the compounds listed in Table 1 were determined using Spartan 14 (Version 1.1.1, WAVEFUNCTION, Inc., Irvine, CA, USA), with a systematic search procedure using molecular mechanics force fields (MMFF). The conformation search was performed with the molecule in a fully relaxed state without any constraints or solvents. The lowest energy conformer (without further optimization) was used to compute the electrostatic surface potential and polar surface area. Exploratory binding site identification and docking to the collagen molecular surface were performed with Schrodinger Sitemap and Glide docking tools, (Schrödinger Drug Discovery Suite 2014-4, Maestro v10.0, Schrödinger, LLC, New York, NY, 2014). PDB xray accession codes 1Q7D and 1DZI were used for modeling purposes.

\section{Statistical Analysis}

Data are expressed as means \pm standard deviation (SD) for each group. Statistics were calculated using SigmaPlot 12.3 (Systat Software, Inc., San Jose, CA USA). Statistical differences were evaluated with ANOVA (followed by post-hoc Dunnett's or Student-Newman-Keuls tests) or t-test depending on the number of groups within an experiment. 


\section{$\underline{\text { RESULTS }}$}

\section{Binding of raloxifene to bone powder}

Fourier transform Infrared (FTIR) spectroscopy was used to assess the retention of raloxifene directly on the bone surface after bone powder was exposed to a solution containing the compound. As topographical features of bone particles could play a role in the surface interaction with small molecules, we assessed various sizes of particles to scale the specific surface area available for the drugbone surface interaction. Decreasing the micro structural heterogeneity of the bone powder is essential to maintain a uniform surface area for surface reactivity studies [21-23]. In addition, the adsorbent properties are functionally dependent on the particle size. Therefore, a seven-point BET (Braunauer, Emmett and Teller Isotherms) was performed to determine the total surface area (TSA) and Specific Surface Area (SSA), of the particles on 4 samples of bone powder representing 4 different particle sizes: $<0.3 \mathrm{~mm},>0.3$ to $<0.53 \mathrm{~mm},>0.53$ to $<0.86 \mathrm{~mm}$, and $>0.86$. The BET analysis revealed that particles smaller than $0.3 \mathrm{~mm}$ had a higher surface area (Supplementary Table 1). Since more exposed surface area can provide more interaction sites for the compound adsorption, the bone powder composed of $<0.3 \mathrm{~mm}$ particles was used for all subsequent studies. Qualitative evaluation of the particles' surface by Scanning Electron Microscopy (SEM) illustrates that micro structural heterogeneity is reduced in smaller sized particles (Supplementary Fig. 1).

To assess binding of raloxifene to bone powder, FTIR spectra were recorded on samples of bone powder ( $<0.3 \mathrm{~mm}$ size) exposed to $20 \mu \mathrm{M}$ raloxifene for $1 \mathrm{~h}$ (Fig. 1). In comparison to the untreated bone powder (Fig. 1A) that showed a broad feature-less spectrum, raloxifene-exposed bone samples showed additional vibration bands in the fingerprint region 1380- $1650 \mathrm{~cm}^{-1}$ (Fig. 1B). Specifically, these new bands were found at 1642, 1560, 1540, 1465, 1384, 1093, 1034, 602 and $564 \mathrm{~cm}^{-1}$. The weak 1642 band likely arose from SP2 C=C stretch. The inhomogeneous broad peak in the region $3434 \mathrm{~cm}^{-1}$ indicated the presence of exchangeable protons while the homogeneous sharper peaks in the fingerprint region pointed to a possible crystalline nature of an adsorbed compound [24-27]. The spectra of a neat solution of $20 \mu \mathrm{M}$ raloxifene without bone powder is shown as reference (Fig. 1C). Although qualitative, the changes in the infrared spectra of the bone powder when incubated with raloxifene suggest that raloxifene is retained on the bone surface. 


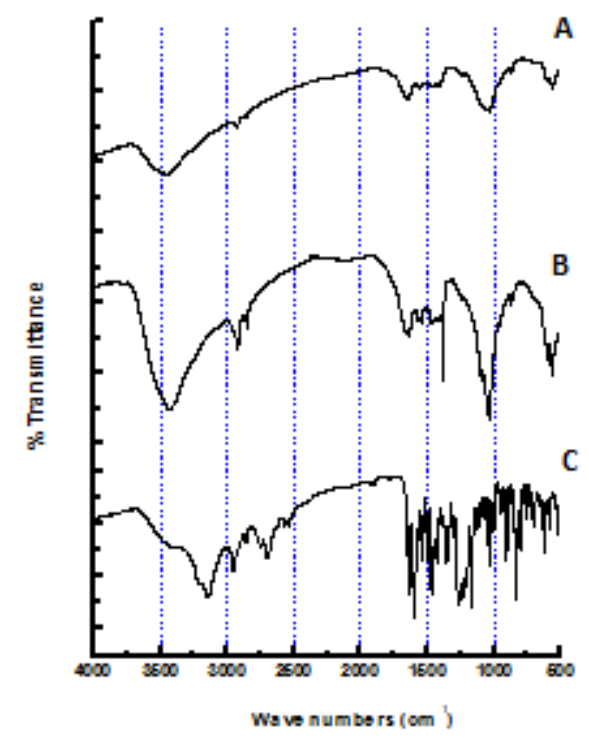

\section{Figure 1}

\section{Analogs for structure-activity studies}

To explore the structural requirements responsible for the potential interaction of raloxifene with bone, a set of raloxifene analogs was chosen in which the central substructure of raloxifene was systematically modified. These analogs are shown in Table 1. Compound 189005 is the methyl ether derivative of raloxifene at the 6-position of the benzothiophene, a structural modification that results in $>100$-fold reduction in the affinity of the molecule for the estrogen receptor [16]; in analog 88074 the basic side chain is absent; 173468 lacks both hydroxyl groups at the 6- and 4'-positions; and compound 153546 is a minimal aryl benzothiophene fragment lacking the entire basic side chain. These modifications systematically impact the lipophilic character of the benzothiophene, as indicated by the differences in the polar surface area (PSA) which is the sum of surfaces of polar atoms (oxygens, nitrogens and hydrogens). The PSA values for this subset of raloxifene analogs spans from $72 \AA^{2}$ (compound 88074) to $24 \AA^{2}$ (compound 173468). Compounds harboring the highest number of $-\mathrm{OH}$ groups were the ones with higher PSA (Table 1). The calculated $\log P$ and $\log D($ Table 1) of this series of compounds corroborate a trend that is consistent with the PSA values.

To determine the effect of these analogs on bone mechanical properties, dog beams (12-15 beams from 4 different dogs) were soaked for 2 weeks with each compound at the concentration of $2 \mu \mathrm{M}$, followed by mechanical testing by 4-point bending. Energy absorption to fracture, the property that best expresses the mechanical effects of raloxifene on bone in these in vitro soaking experiments, was $25 \%$ higher in beams that were treated with 189005 compared to DMSO-soaked control beams (Table 1). 
The remaining 6 compounds elicited an effect that was either equivalent to or lower than DMSO controls.

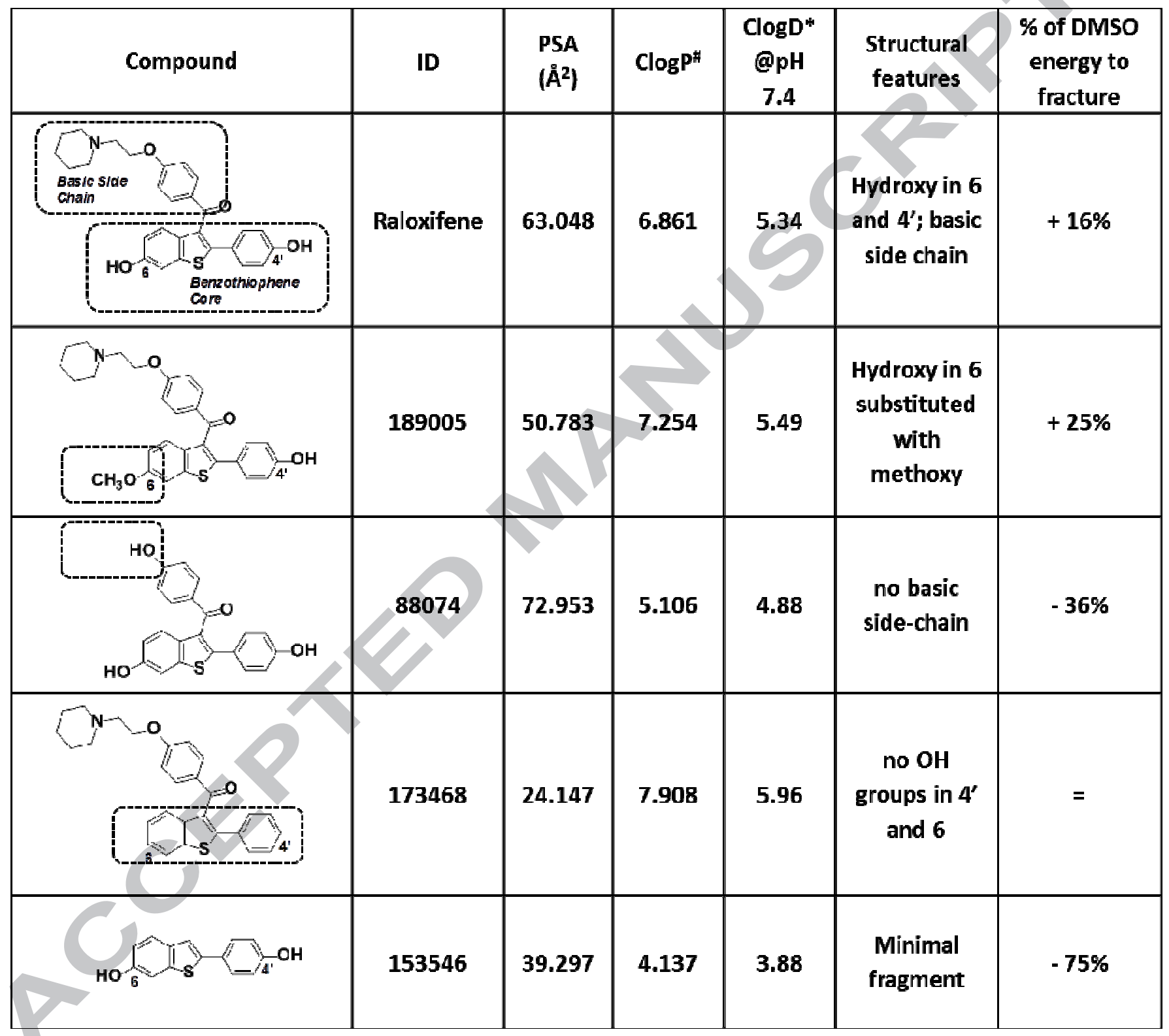

Table 1 


\section{Binding of raloxifene analogs to bone powder, collagen and hydroxyapatite}

NMR spectroscopy was used to quantify the amount of compound remaining in solution after incubation with bone powder and the primary component of bone, HAP or type I collagen. In this assay, if interaction occurs, the amount of compound retrieved from the supernatants after incubation with bone is diminished, an indication that some of the compound is retained in the matrix.

The ability of raloxifene to interact with bone powder was first tested by solution state ${ }^{1} H$ NMR. In addition to raloxifene, compounds 88074 and 189005 were tested to compare the relative contributions of the hydroxyl groups (e.g. in 189005) and of the side chain (e.g. in 88074) to bone binding. Incubation of $50 \mu \mathrm{M}$ raloxifene with $10 \mathrm{mg}$ of bone powder for $1 \mathrm{~h}, 4 \mathrm{~h}$ and $8 \mathrm{~h}$ resulted in a statistically significant decrease of raloxifene signal in the recovered supernatants compared to the signal present in control solution that has not been exposed to bone powder (Fig. 2A). These results indicate retention of raloxifene by the bone sample. The methoxy analog of raloxifene, 189005, bound in a similar manner to raloxifene, with the signal almost completely disappeared from the supernatant (Fig. $2 \mathrm{C}$ ). In contrast, the analog lacking the basic side chain (88074) also had reduced signal in the supernatant, but the magnitude of the signal intensity drop was lower compared to that observed for raloxifene (Fig. 2B). 
A

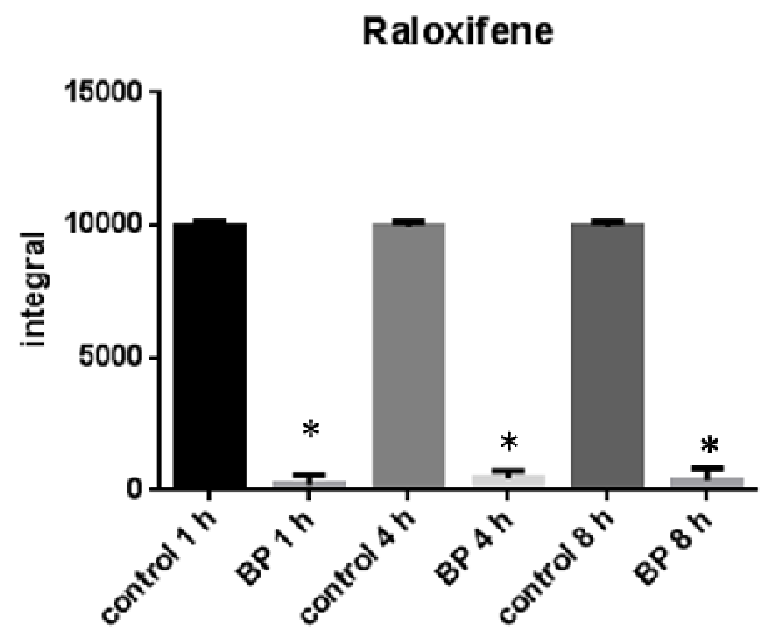

B

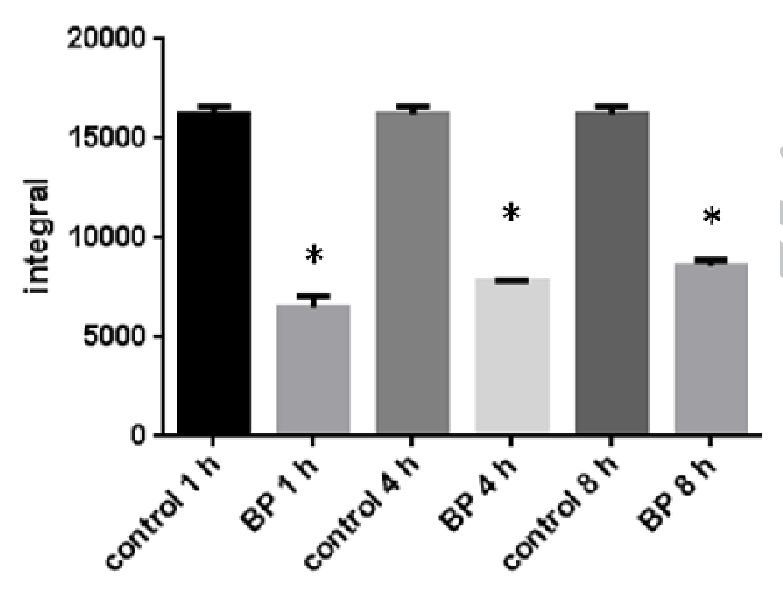

C

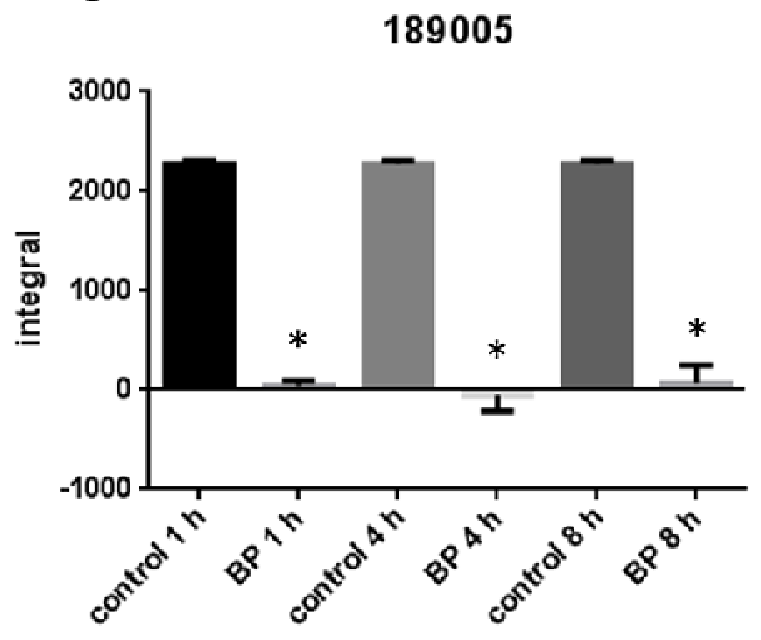

\section{Figure 2}

To assess whether the organic constituent of bone might be retaining these compounds, we incubated $50 \mu \mathrm{M}$ of raloxifene with a commercially available tri-dimensional collagen matrix composed exclusively of collagen 1a1. Incubation with the collagen matrix for $1 \mathrm{~h}, 4 \mathrm{~h}$ or $8 \mathrm{~h}$ resulted in a statistically significant reduction in the NMR signals of raloxifene compared to the control experiment, indicating binding of raloxifene to the collagen matrix (Fig. 3A). A similar result was observed in compound 189005 (Fig 3B). On the other hand, the interaction of 88074 with collagen, although significant (Fig 3C), was less than the interaction found with the other 2 compounds. 
A

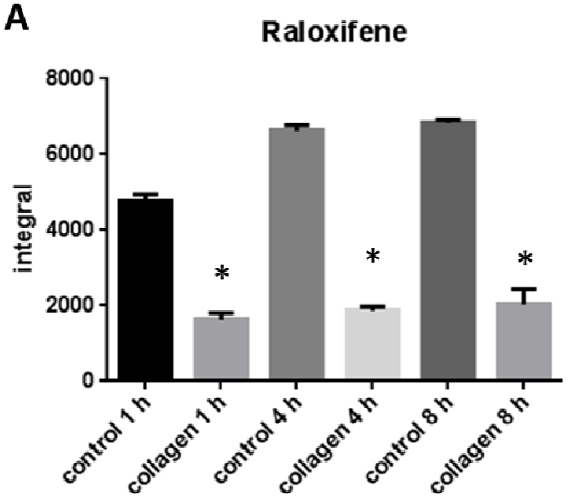

C

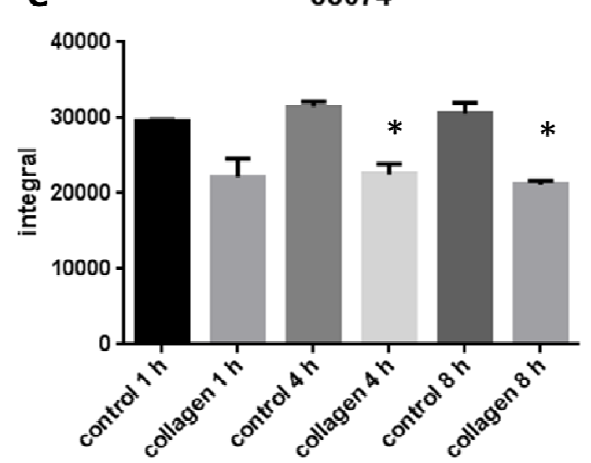

B

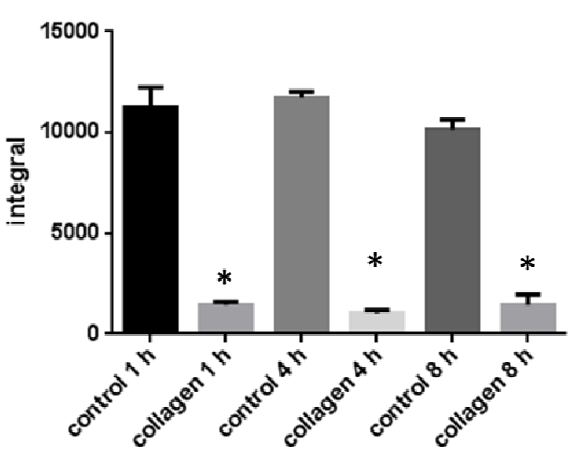

D

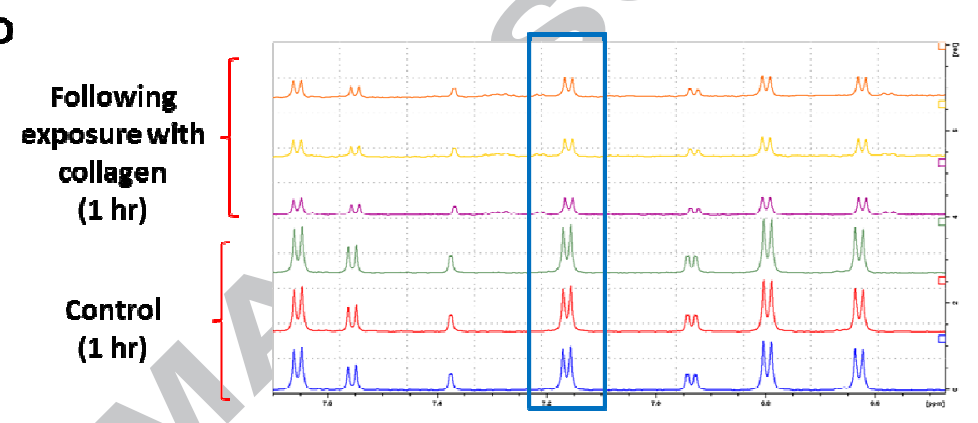

Figure 3

We then explored the bone binding properties of an array of direct analogs of raloxifene in order to investigate the specific structural features responsible for its unique activity (Fig. 4 and Table 2). In this set of experiments we also included incubations with the mineral component of bone, hydroxyapatite (HAP), which contributes $95 \%$ of crystallinity to the mineral, in order to examine the surface interactions between HAP and the raloxifene analogs. Exposure of raloxifene to bone powder resulted in a $54.2 \%$ reduction in signal after $1 \mathrm{~h}$ and $99.0 \%$ reduction after $16 \mathrm{~h}$ of incubation (Table 2). On the other hand, incubation with hydroxyapatite did not elicit a decrease, but rather, it resulted in a unexpected increase in the amount of raloxifene recovered from the supernatants (Fig. 4); we hypothesize that this increase is due to an improvement in raloxifene solubility facilitated by the very charged HAP. Compound 88074 was also reduced after incubation with bone powder, but to a lesser extent than raloxifene (by $26.4 \%$ at $1 \mathrm{~h}$ and $34.1 \%$ at $16 \mathrm{~h}$ ), while the reduction with HAP was minimal (13.3\% and $15.1 \%$ at $1 \mathrm{~h}$ and $16 \mathrm{~h}$ respectively).

To gain a better understanding of the role played by the hydroxyl $(\mathrm{OH})$ groups in the interaction with the bone samples, we investigated three additional compounds: 173468, which lacks hydroxyl groups at the 4'- and 6-positions, 153546, which comprises only the aryl benzothiophene core, and 17b-estradiol. The 
signal from compound 173468 was not reduced after $1 \mathrm{~h}$ of incubation with bone powder, whereas it was decreased by about $89 \%$ after $16 \mathrm{~h}$, while exposure to HAP did not elicit a significant reduction in the recovered signal. Interestingly, the minimal fragment 153546 showed only a small ability to be retained by bone powder, with a $42.8 \%$ and $46.4 \%$ reduction in signal after $1 \mathrm{~h}$ and $16 \mathrm{~h}$ in the presence of bone powder (Fig. 4 and Table 2). Moreover, we included incubations of HAP and bone powder with Alendronate, a well-known bisphosphonate that was shown to bind avidly to both matrices. The comparison between the percent drop in NMR signal with each compound and Alendronate in the presence of bone powder reveals that Raloxifene binds as avidly as Alendronate to bone powder. In addition, while Alendronate is completely sequestered by HAP at $16 \mathrm{~h}$, incubations of raloxifene with HAP did not show any decrease, further confirming that the ability of raloxifene to bind bone powder relies on its interaction with the organic portion of the bone matrix and not with the mineral phase. The interaction of raloxifene analogs with bone powder is specific, as shown by the lack of NMR signal drop in supernatants containing the compound 3103672, a GPCR agonist with sufficient structural diversity from raloxifene to be used as an appropriate negative control (Fig. 4 and Table 2).

A
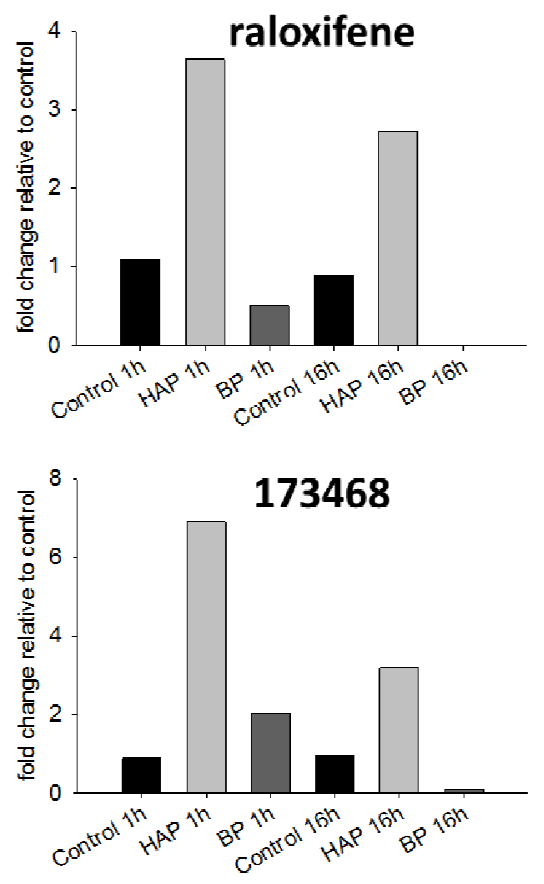
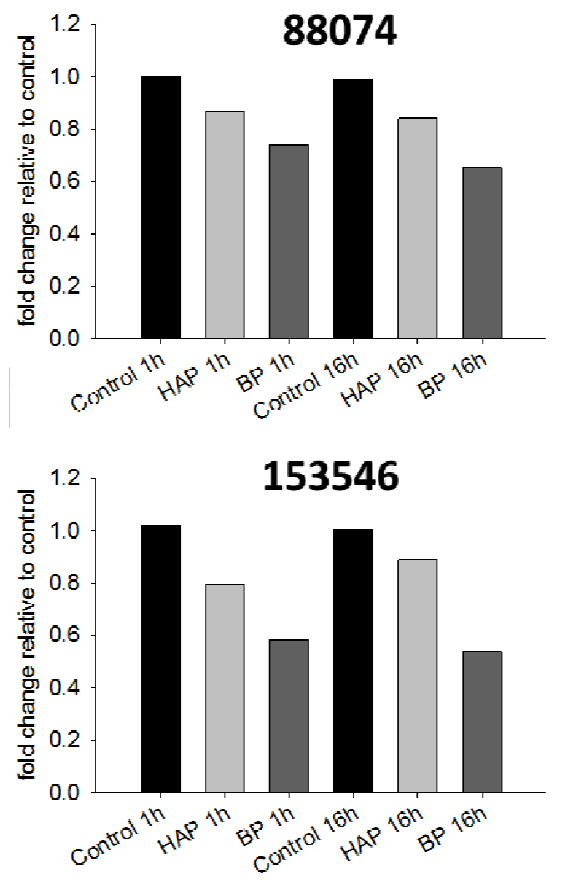

B
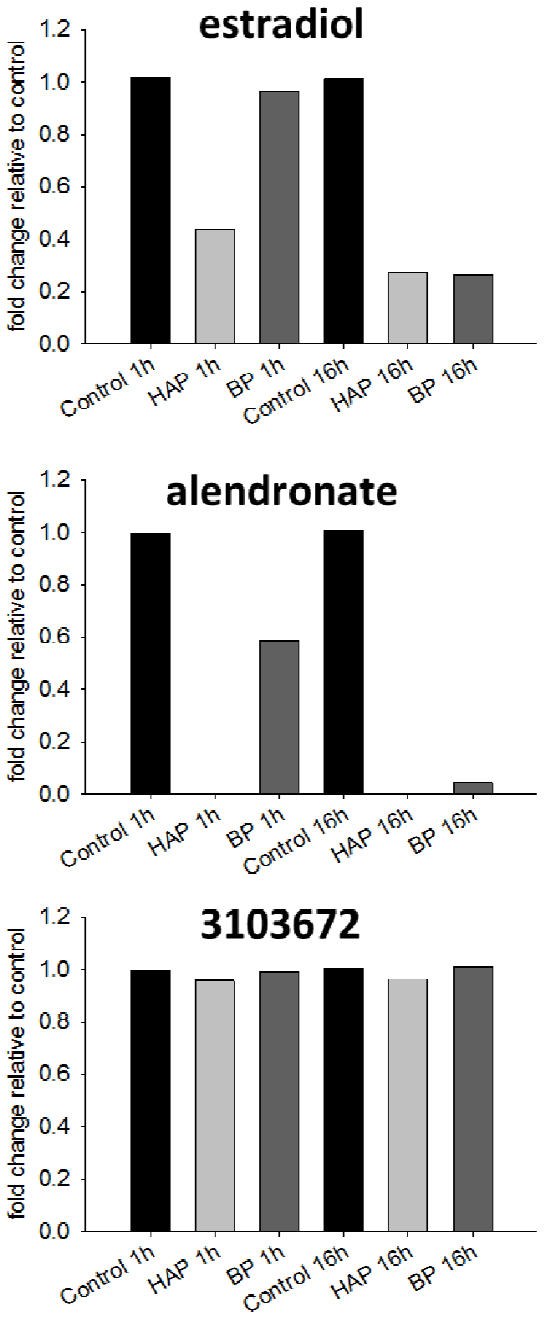

Figure 4 


\begin{tabular}{|c|c|c|c|c|c|}
\hline$\%$ decrease relative to control & HAP $1 \mathrm{~h}$ & BP $1 \mathrm{~h}$ & $\%$ decrease relative to control & HAP $16 h$ & BP $16 h$ \\
\hline Raloxifene & -231.7 & 54.2 & Raloxifene & -206.1 & 99.0 \\
\hline 88074 & 13.3 & 26.4 & 88074 & 15.1 & 34.1 \\
\hline 173468 & -422.5 & -132.4 & 173468 & -237.2 & 89.0 \\
\hline 153546 & 22.3 & 42.8 & 153546 & 11.7 & 46.4 \\
\hline 189005 & -57.9 & 21.0 & 189005 & -286.2 & 73.9 \\
\hline estradiol & 57.1 & 5.3 & estradiol & 73.0 & 73.9 \\
\hline alendronate & 100.1 & 41.1 & alend ronate & 99.7 & 95.6 \\
\hline 3103672 & 3.9 & 0.3 & 3103672 & 4.1 & -0.5 \\
\hline $\begin{array}{c}\% \text { decrease compared to } \\
\text { Alendronate }\end{array}$ & HAP $1 \mathrm{~h}$ & BP $1 \mathrm{~h}$ & $\%$ decrease compared to Alendronate & HAP $16 h$ & BP 16h \\
\hline Raloxifene & -231.7 & 54.2 & Raloxifene & -206.8 & 103.6 \\
\hline 88074 & 13.3 & 26.4 & 88074 & 15.1 & 35.6 \\
\hline 173468 & -422.5 & -132.4 & 173468 & -238.0 & 93.1 \\
\hline 153546 & 22.3 & 42.8 & 153546 & 11.7 & 48.6 \\
\hline 189005 & -57.9 & 21.0 & 189005 & -287.2 & 77.4 \\
\hline estradiol & 57.1 & 5.3 & estradiol & 73.2 & 77.4 \\
\hline alendronate & 100 & 100 & alendronate & 100 & 100 \\
\hline 3103672 & 3.9 & 0.3 & 3103672 & 4.1 & -0.5 \\
\hline
\end{tabular}

Table 2

\section{DISCUSSION AND CONCLUSIONS}

We hypothesized that the binding profile to bone matrix and its components may shed light into the mechanism of action of raloxifene on osteoporosis pharmacology. The analogs we investigated are a systematic truncation of the parent structure in order to provide a broad range of structural diversity within the scaffold. It included variations in the phenol groups on the benzothiophene core, in order to rule out ER-mediated events, and modifications of the basic side chain. The results indicate that benzothiophene-derived SERMs can interact with bone matrix in a structure-dependent manner. Moreover, this interaction appears to be only with the organic component or the organic/mineral composite, as it does not exist with pure hydroxyapatite.

Previous studies have established that the structural features critical for the ER mediated pharmacology of raloxifene are the hydroxyl substituent at the 6- and, to a lesser extent, the 4'-positions of the benzothiopene core. Structure activity relationship (SAR) studies have shown that raloxifene-like 
compounds where the 6- and/or 4'-hydroxyl groups are absent or are capped as methyl ethers show a several fold decrease in the relative binding affinity to the ER $[16,17]$.

Compared to the essential role played by the hydroxyl groups $(-\mathrm{OH})$ in binding the ER, it appears that they play an attenuated role in modulating the physical interactions with bone. For example, compound 173468 lacks the $-\mathrm{OH}$ groups on the 6- and 4'-positions and has a Ki to the ER's of $>10 \mu \mathrm{M}$. In comparison, raloxifene binds with a Ki of $0.43 \mathrm{nM}$ to ERalpha and $4.3 \mathrm{nM}$ to ERbeta. Yet compound 173468 is shown here to retain bone matrix binding activity, with a magnitude comparable to that of raloxifene. In contrast, compound 153546 binds with high affinity to ER alpha and beta with Ki's of 4.1 and $2.3 \mathrm{nM}$, respectively, yet demonstrates very low binding to bone powder or HAP (Fig. 4). Taken together, these data indicate that the structural requirements for bone matrix binding differ from the ones needed to bind the estrogen receptor. However, the phenolic groups may be responsible for a more robust binding of the compound to the bone powder that allows greater binding at the earlier time point. Thus, while raloxifene signal decreases by $54.2 \%$ already after $1 \mathrm{~h}$ of incubation with bone powder, 173468 signal does not change, although the two compounds display a comparable decrease at $16 \mathrm{~h}$.

The basic side-chain appears to be a crucial structural requirement for bone matrix binding. This is illustrated by SAR studies of the basic side chain in which portions of this component have been sequentially truncated. Removal of the aminoethylpiperdine moiety, as in 88074 , reduces bone binding, compared to raloxifene. Further truncation of the entire aroyl side chain, as in 153546, also results in reduced interaction of the compound with bone powder (Fig. 4). The importance of the basic side chain was further assessed using the molecular modeling software SiteMap (SiteMap, version 3.0, Schrodinger, LLC, New York, NY, 2014) and crystallographic structures (PDB accession codes 1Q7D and 1DZI) to search for potential binding sites on the surface of collagen. The sitemap obtained for both the triple fibril helix and collagen complexed with an integrin indicated a groove with sufficient space to accommodate raloxifene analogs. This groove is rich in acidic glutamate residues which is consistent with affinity for the basic side chain. A high resolution model image of a collagen triple chain fiber molecular surface (Fig. 5, top panel) shows the putative binding site as small white spheres. A second closer view (Fig. 5, bottom panel) shows 189005 docked from two different angles. This pose highlights two polar interactions, the piperidine basic- $\mathrm{N}$ interacting with E12 of orange chain and the pendant phenolic $\mathrm{OH}$ interacting with E12 on blue chain. The benzothiophene core is stacking with F9 (blue chain) in a hydrophobic interaction. It is also proposed that the benzothiophene could have a second hydrophobic interaction with an adjacent triple chain fiber. Thus, in this model, there are important polar interactions between raloxifene and one triple chain collagen fiber (basic $\mathrm{N}$ and phenol $\mathrm{OH}$ ) but additionally hydrophobic interactions between the benzothiophene ring and F9 and possibly other collagen triple chain fibers in adjacent positions. 

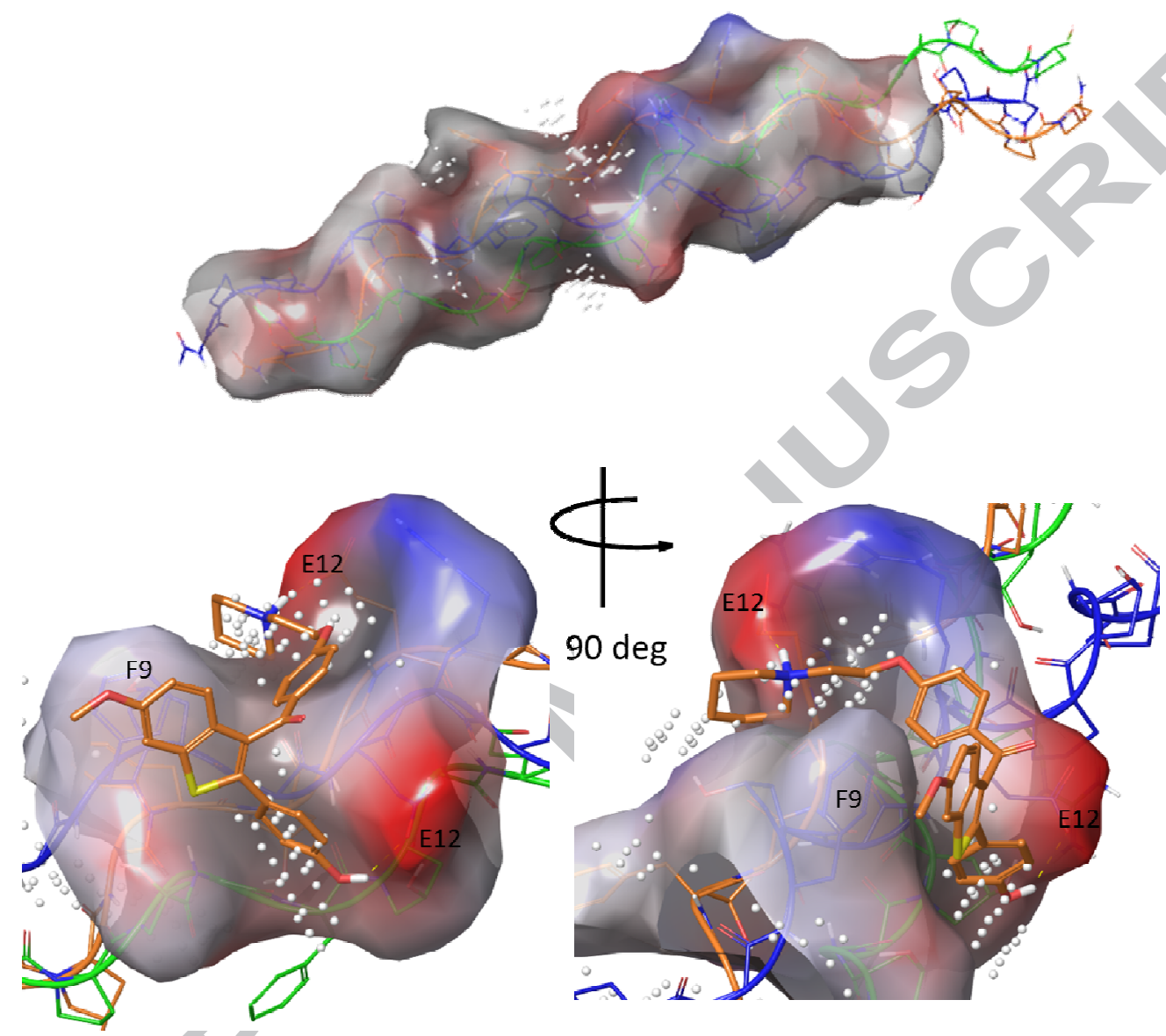

\section{Figure 5}

At the molecular level, raloxifene and the analogs evaluated in this study could interact with bone matrix through two types of surface interactions: i) hydrophilic or electrostatic interactions via the piperidine nitrogen, and/or ii) hydrophobic interactions (van der Waals) via the aliphatic carbons and aromatic rings of the benzothiophene core, the tether to the basic nitrogen, and the methylene groups of the piperidine ring. Our finding that the side chain is required for bone matrix binding could be explained by a combination of electrostatic interactions by the piperidine nitrogen and of an important role of the hydrophobic regions. Compound 189005, having the lowest PSA (50.8 $\AA$ ), cLogP (7.254) and cLogD (5.49) (Table 1), and hence a greatest proportion of hydrophobic surface, supports this hypothesis, as it was retained by bone powder and collagen with highest efficiency. The hydrophobic regions (green colored) of raloxifene and 189005 are large and extend throughout the molecule (Fig. 6). 

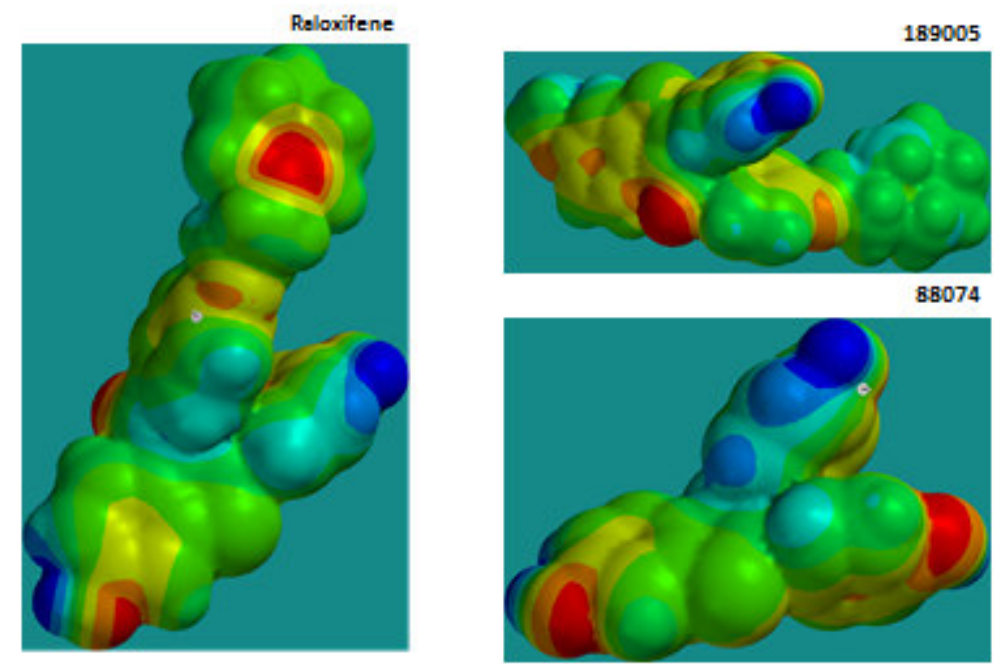

\section{Figure 6}

In contrast, 88074 has a higher PSA (72.9 $\AA$ ), cLogP (5.106), and $\operatorname{cLogD}(4.88)$. It contains no basic side chain and has fewer aliphatic groups than 189005. Thus, it is significantly less hydrophobic and correspondingly less able to take advantage of both the electrostatic interactions imparted by the basic nitrogen and the van der Waals contacts with the organic phases of the bone matrix. This reduced hydrophobicity is consistent with the reduced bone and collagen binding when comparing 88074 to 189005. Our data indicate that the interaction of the raloxifene analogs with bone is due to a combination of intrinsic structural features. The basic nitrogen in the piperidine side chain appears to be the dominant feature. However, the hydrophobic regions and the $-\mathrm{OH}$ groups play an important role as well. The interaction of compounds with the bone surface has historically been predominantly electrostatic, as in the case of tetracyclines, calcein, and bisphosphonates, and has involved the mineral phase rather than the organic phase. Bisphosphonates, in particular, have been shown to bind avidly to HAP using a NMR assay similar to the one used in this paper [20]. However, our data suggest that the hydrophobic interactions are potentially another important way to induce greater molecule-surface affinity and that they occur through the organic phase (especially collagen) rather than the hydroxyapatite.

To determine if the bone binding data correlated to a functional effect on bone strength, we tested raloxifene, 88074 and 189005 for their ability to affect bone material toughness using an ex-vivo methodology previously described [15]. Soaking of dog bone beams in raloxifene and 189005 resulted in a similar enhancement in toughness while 88074 did not elicit an increase (Table 1). These data are consistent with the bone binding data for these compounds.

The biophysical techniques described in this manuscript allowed us to determine the previously unknown ability of raloxifene to bind bone matrix. However, the experimental methodology cannot 
distinguish between passive, non-specific compound deposition (or retention), and specific binding to the tested surface. Moreover, the 3D collagen fibrillar matrix employed in our experiments does not reproduce the conformation of the collagen fibers in bone and does not allow a direct comparison of the amount of collagen present in the 3D matrix with that present in the bone powder.

Taken together, the data provide evidence that raloxifene, and select analogs, have the ability to bind bone matrix in an unexpected fashion. Raloxifene does not effectively interact with the mineral phase of bone, but rather interacts with the organic portion potentially through hydrophobic interactions. We propose that the binding of benzothiophene SERMs to bone depends on the combination of the hydroxyl groups at the 6- and 4'- positions, the basic amino side-chain and the intrinsic aliphatic character. Of these groups, the basic amino group is the primary determinant of bone binding. Given that other bone active SERMs, such as tamoxifen, contain a basic side chain, it would be interesting to determine if it also affects bone toughness. Additional studies are required to clarify if these structural motifs could be transferable to generate new chemical entities for the improvement of bone mechanical properties.

\section{Acknowledgements}

All the experiments were funded by Eli Lilly \& Co. The authors thank Drew Brown for making bone beams, bone powder, and performing all soaking experiments.

\section{Figure and Table legends}

Figure 1. Detection of adsorbed raloxifene on the bone powder by FTIR. In comparison to bone powder that has never been in contact with raloxifene (A), bone exposed to $50 \mu \mathrm{M}$ raloxifene for $1 \mathrm{~h}$ shows rich fingerprint region at $1380-1650 \mathrm{~cm}-1$ (B). Incubation with raloxifene results on the appearance of new sharper bands at 1642, 1560, 1540, 1465, 1384, 1093, 1034, 602 and $564 \mathrm{~cm}-1$ similar to the bands in the neat spectrum raloxifene alone (C). The sharper peaks in B reflect crystalline nature of the adsorbed species as against the broad (hence glassy) bands observed in the bone powder matrix (C).

Table 1. Tested compounds and their structural features. Polar Surface Area (PSA) is calculated from the lowest energy conformation, (using MMFF). cLogP is from BioByte, (Claremont, CA). cLogD@pH 7.4 is from ChemAxon, (Cambridge, MA). Red boxes enclose critical structural features of the compounds.

Figure 2. Detection and relative quantification of raloxifene and its analogs in supernatants after incubation with bone powder by NMR. $1 \mathrm{H}$ NMR spectra (0-8 ppm range) of $50 \mu \mathrm{M}$ raloxifene (A), 88074 (B) and 189005 (C) incubated with and without $10 \mathrm{mg}$ of dog bone powder for $1 \mathrm{~h}, 4 \mathrm{~h}$ or $8 \mathrm{~h}$. Graphs represent the integral of the peaks. Bars are mean \pm s.d. $N=3 ;{ }^{*} p<0.05$ by t-test vs control at the same time point. BP: bone powder 
Figure 3. Detection and relative quantification of raloxifene and its analogs in supernatants after incubation with collagen by NMR. (A) $1 \mathrm{H}$ NMR spectra (0-8 ppm range) of $50 \mu \mathrm{M}$ raloxifene. NMR spectra of 189005 (B) and 88074 (C) incubated with and without $0.5 \mathrm{ml}$ of polymerized 3D collagen matrix. Graphs represent the integral of the peaks. Bars are mean $\pm \mathrm{s} . \mathrm{d}$. N=3; ${ }^{*} p<0.05$ by t-test vs control at the same time point. (D) Representative NMR spectra of a solution of raloxifene after incubation with collagen (top three) and a control solution (bottom three) that highlights the marked difference in the height of the peaks between the two conditions. Each spectrum represents a supernatant obtained from separate incubations.

Figure 4. Detection and quantification of raloxifene and its analogs in supernatants after incubation with bone powder by NMR. Quantification of residual raloxifene, 88074, 173468, 153546, 17beta-estradiol, alendronate and 3103672 after incubation of a $50 \mu \mathrm{M}$ solution of each compound with $10 \mathrm{mg}$ of bone powder and $5 \mathrm{mg}$ of HAP for $1 \mathrm{~h}$ or $16 \mathrm{~h}$. Bars represent fold change relative to control. HAP: hydroxyapatite; BP: bone powder. $\mathrm{N}=2$.

Table 2. Results presented in Figure 4, expressed as \% decrease relative to control (top portion of the table) and \% decrease relative to alendronate (bottom portion). HAP: hydroxyapatite; BP: bone powder.

Figure 5. Top panel: collagen triple chain fiber molecular surface with putative binding site (near the center) indicated with small white spheres, surface colored by underlying atom-type. PDB code= 1Q7D. Bottom panel: two views turned 90 degrees from each other, show 189005 docked to the putative site, showing two polar interactions, the piperidine basic- $\mathrm{N}$ interacting with E12 of orange chain and the pendant phenolic $\mathrm{OH}$ interacting with $\mathrm{E} 12$ on blue chain. The benzothiophene core is stacking with F9 (blue chain).

Figure 6. Electrostatic Potential on an Electron Density Isosurface corresponding to a van der Waals Surface of raloxifene, 88074 and 189005. Representation of the hydrophobic regions (green colored).

Supplementary Table 1. Characterization of bone powder particles. BET SSA: Specific Surface Area, measured by Braunauer, Emmett and Teller (BET) Isotherms.

Supplementary Figure 1. Representative images of bone powder preparations obtained by SEM. Images were collected at 50X. 


\section{REFERENCES}

1. Rey, J. R.; Cervino, E. V.; Rentero, M. L.; Crespo, E. C.; Alvaro, A. O.; Casillas, M. Raloxifene: mechanism of action, effects on bone tissue, and applicability in clinical traumatology practice. The open orthopaedics journal 2009, 3, 14-21.

2. Khajuria, D. K.; Razdan, R.; Mahapatra, D. R. Drugs for the management of osteoporosis: a review. Revista brasileira de reumatologia 2011, 51, 365-371, 379-382.

3. Bryant, H. U. Mechanism of action and preclinical profile of raloxifene, a selective estrogen receptor modulation. Reviews in endocrine \& metabolic disorders 2001, 2, 129-138.

4. Carneiro, A. L.; de Cassia de Maio Dardes, R.; Haidar, M. A. Estrogens plus raloxifene on endometrial safety and menopausal symptoms--semisystematic review. Menopause 2012, 19, 830-834.

5. Gizzo, S.; Saccardi, C.; Patrelli, T. S.; Berretta, R.; Capobianco, G.; Di Gangi, S.; Vacilotto, A.; Bertocco, A.; Noventa, M.; Ancona, E.; D'Antona, D.; Nardelli, G. B. Update on raloxifene: mechanism of action, clinical efficacy, adverse effects, and contraindications. Obstetrical \& gynecological survey 2013, 68, 467-481.

6. Lee, W. L.; Chao, H. T.; Cheng, M. H.; Wang, P. H. Rationale for using raloxifene to prevent both osteoporosis and breast cancer in postmenopausal women. Maturitas 2008, 60, 92-107

7. Cosman, F. Selective estrogen-receptor modulators. Clinics in geriatric medicine 2003, 19, 371$\underline{379}$

8. Martino, S.; Disch, D.; Dowsett, S. A.; Keech, C. A.; Mershon, J. L. Safety assessment of raloxifene over eight years in a clinical trial setting. Current medical research and opinion 2005, 21, 14411452

9. Das, S.; Crockett, J. C. Osteoporosis - a current view of pharmacological prevention and treatment. Drug design, development and therapy 2013, 7, 435-448

10. Riggs, B. L.; Melton, L. J., 3rd. Bone turnover matters: the raloxifene treatment paradox of dramatic decreases in vertebral fractures without commensurate increases in bone density. Journal of bone and mineral research 2002, 17, 11-14

11. Sarkar, S.; Mitlak, B. H.; Wong, M.; Stock, J. L.; Black, D. M.; Harper, K. D. Relationships between bone mineral density and incident vertebral fracture risk with raloxifene therapy. Journal of bone and mineral research 2002, 17, 1-10

12. Aref, M.; Gallant, M. A.; Organ, J. M.; Wallace, J. M.; Newman, C. L.; Burr, D. B.; Brown, D. M.; Allen, M. R. In vivo reference point indentation reveals positive effects of raloxifene on mechanical properties following 6 months of treatment in skeletally mature beagle dogs. Bone 2013, 56, 449-453

13. Allen, M. R.; Hogan, H. A.; Hobbs, W. A.; Koivuniemi, A. S.; Koivuniemi, M. C.; Burr, D. B. Raloxifene enhances material-level mechanical properties of femoral cortical and trabecular bone. Endocrinology 2007, 148, 3908-3913

14. Allen, M. R.; Iwata, K.; Sato, M.; Burr, D. B. Raloxifene enhances vertebral mechanical properties independent of bone density. Bone 2006, 39, 1130-1135

15. Gallant, M. A.; Brown, D. M.; Hammond, M.; Wallace, J. M.; Du, J.; Deymier-Black, A. C.; Almer, J. D.; Stock, S. R.; Allen, M. R.; Burr, D. B. Bone cell-independent benefits of raloxifene on the skeleton: a novel mechanism for improving bone material properties. Bone 2014, 61, 191-200

16. Grese, T. A.; Sluka, J. P.; Bryant, H. U.; Cullinan, G. J.; Glasebrook, A. L.; Jones, C. D.; Matsumoto, K.; Palkowitz, A. D.; Sato, M.; Termine, J. D.; Winter, M. A.; Yang, N. N.; Dodge, J. A. Molecular 
determinants of tissue selectivity in estrogen receptor modulators. Proceedings of the National Academy of Sciences of the United States of America 1997, 94, 14105-14110

17. Grese, T. A.; Cho, S.; Finley, D. R.; Godfrey, A. G.; Jones, C. D.; Lugar, C. W. $3^{\text {rd }}$; Martin, M. J.; Matsumoto, K.; Pennington, L. D.; Winter, M. A.; Adrian, M. D.; Cole, H. W.; Magee, D. E.; Phillips, D. L.; Rowley, E. R.; Short, L. L.; Glasebrook, A. L.; Bryant, H. U. Structure-activity relationships of selective estrogen receptor modulators: modifications to the 2arylbenzothiophene core of raloxifene. Journal of medicinal chemistry 1997, 40, 146-167

18. Hong, Y.; Wang, Z.; Jin, X. Sulfuric acid intercalated graphite oxide for graphene preparation. Scientific reports 2013, 3, 3439

19. Singh, J.; Kumar, P.; Hui, K.S.; Hui, K.N.; Ramam, K.; Tiwari, R.S.; Srivastava, O.N. Synthesis, bandgap tuning, structural and optical investigations of $\mathrm{Mg}$ doped $\mathrm{ZnO}$ nanowires. CrystEngComm 2012, 14

20. Jahnke, W.; Henry, C. An in vitro assay to measure targeted drug delivery to bone mineral. ChemMedChem 2010, 5, 770-776

21. Carpenter, F. G.; Deitz, V.R. Methods of Sieve Analysis With Particular Reference to Bone Char. Journal of Research of the National Bureau of Standards 1950, 45

22. Cai, K.; Frant, M.; Bossert, J.; Hildebrand, G.; Liefeith, K.; Jandt, K. D. Surface functionalized titanium thin films: zeta-potential, protein adsorption and cell proliferation. Colloids and surfaces. B, Biointerfaces 2006, 50, 1-8

23. Dufrene, Y. F.; Marchal, T.G.; Rouxhet, P.G. Influence of substratum surface properties on the organization of adsorbed collagen films: in situ characterization by atomic force microscopy. Langmuir 1999, 15

24. Rehman, I.; Bonfield, W. Characterization of hydroxyapatite and carbonated apatite by photo acoustic FTIR spectroscopy. Journal of materials science. Materials in medicine 1997, 8, 1-4

25. Errassifi, F.; Sarda, S.; Barroug, A.; Legrouri, A.; Sfihi, H.; Rey, C. Infrared, Raman and NMR investigations of risedronate adsorption on nanocrystalline apatites. Journal of colloid and interface science 2014, 420, 101-111

26. Patil, P. H.; Belgamwar, V.S.; Patil, P.R.; Surana, S.J. Solubility Enhancement of Raloxifene Using Inclusion Complexes and Cogrinding Method. Journal of Pharmaceutics 2013 URL: http://dx.doi.org/10.1155/2013/527380

27. Takata, S.; Shibata, A.; Yonezu, H.; Yamada, T.; Takahashi, M.; Abbaspour, A.; Yasui, N. Biophysic evaluation of bone quality-application of Fourier transform infrared spectroscopy and phosphorus-31 solid-state nuclear magnetic resonance spectroscopy. The journal of medical investigation 2004, 51, 133-138 

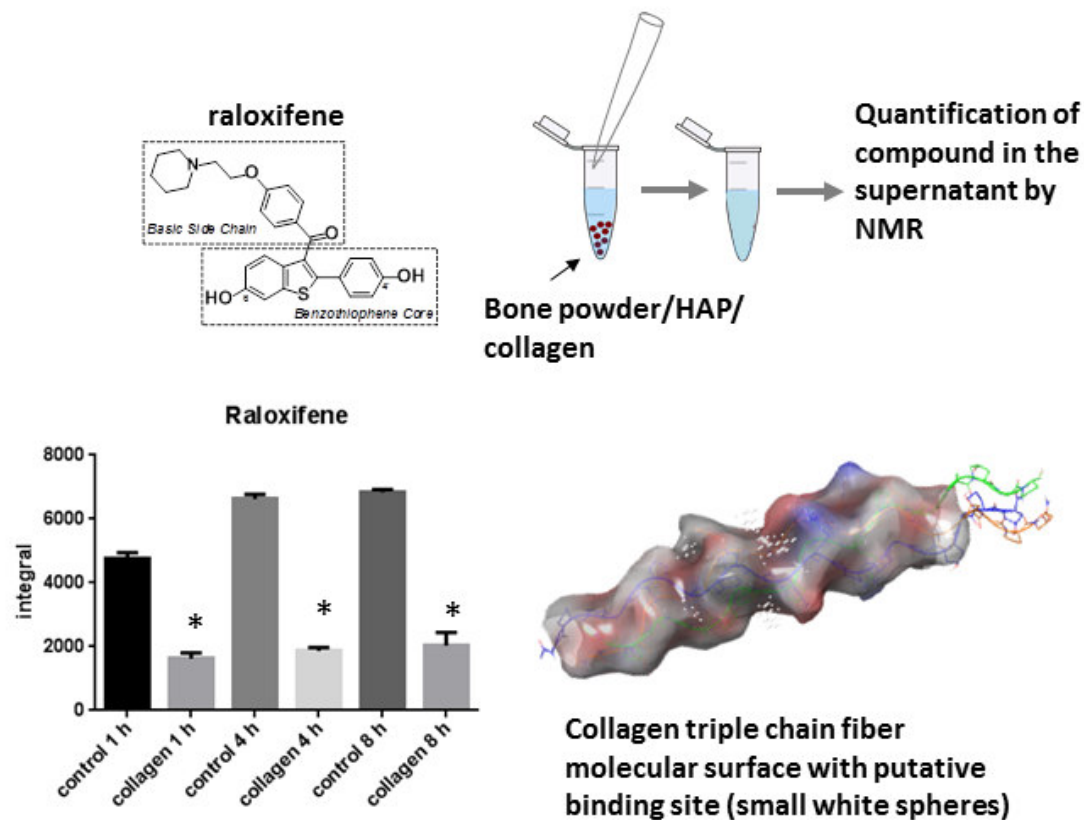

Collagen triple chain fiber molecular surface with putative binding site (small white spheres) 